\title{
From tips to domes
} \section{PANalytica}

DHS 900 www.panalytical.com

High-temperature X-ray diffraction

PANalytical introduce a new heating attachment, the Anton Paar DHS 900, a 'domed hot stage', for fitting to its X'Pert PRO MRD X-ray diffractometer. XRD applications such as rocking curve analysis, in-plane diffraction, wafer mapping, reciprocal space mapping and reflectivity measurements, and stress and texture analysis can now be carried out at temperatures of up to $900^{\circ} \mathrm{C}$, including the combined movements of several axes (such as performed when recording complete pole figures with additional oscillation). The DHS 900 is easily mounted onto the MRD cradle, and is fitted with a dome that is transparent for X-ray diffraction. This allows samples to be investigated in vacuum, air or inert gas, thus avoiding oxidation or other chemical reactions at elevated temperatures. The dome and the base plate of the heating attachment are cooled using compressed air to ensure that the console in which the diffractometer is placed is not unduly heated up during measurements, and stays within its temperature limits for normal operation.

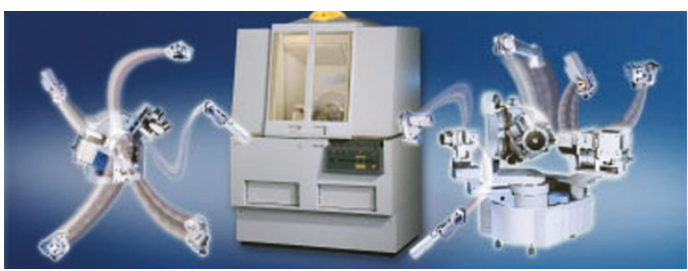

\section{nature materials}

The 'New on the Market' section of Nature Materials is designed to give our readers access to the latest technologies, products and services available in the highly competitive materials science and engineering fields. With a worldwide circulation, Nature Materials is an invaluable resource for all scientists, in both academia and industry, who are active in the process of discovering and developing materials and materials-related concepts. We would like to invite you to send your Press Release Information about your new technologies, products and services for FREE inclusion in the feature section 'New on the Market'.

Weblinks will accompany each PR and some pictures will also be included. As an editorial page of Nature Materials, these PRs will also appear online.

In order to be considered for inclusion, we require press information of between 100 and 250 words in English detailing your product, technology or service. Inclusion of photographs is optional but preferred.

For further information please contact Gerard Preston, Senior European Advertising Manager atg.preston@nature.com

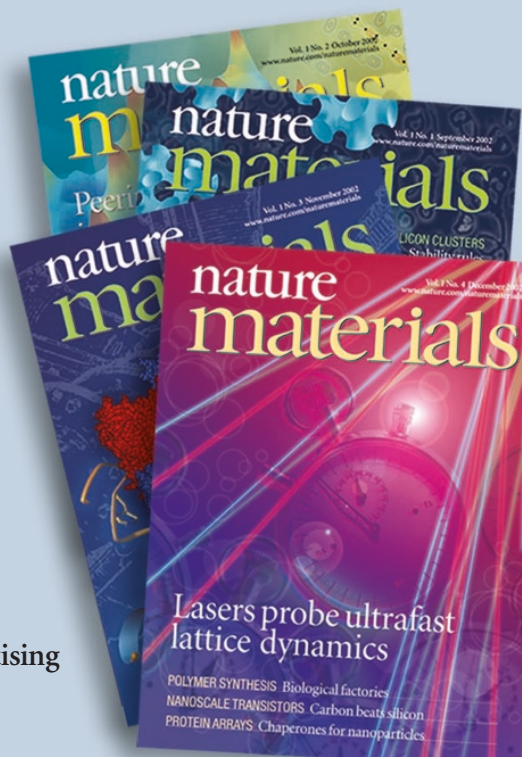

OIM 2000, 4000, 7000 EDAX wuw.edax.com

Electron backscatter diffraction

EDAX are introducing a 'next generation' of electron backscatter diffraction (EBSD) systems, the OIM 2000, OIM 4000 and OIM 7000. These are designed to meet the needs of a wide variety of users, from introductory analytical packages to high-end advanced research-level systems. They include state-ofthe-art EBSD cameras,

\section{PROBEMAX}

\section{SEM of carbon nanotube tips}

nPoint are now providing scanning electron microscope images of their carbon nanotube (CNT) tips, which will allow the user to select the appropriate probe for their atomic force microscope application. CNT tips provide accurate high-resolution imaging of deep surface features-a great advantage in the semiconductor market. The ProbeMax carbon nanotube probes consist of multiwall CNTs or nanotube bundles, mounted onto commercially available silicon atomic force microscope probes. They offer long lifetime and the capability of measuring high-aspectratio features. These probes can replace conventional silicon

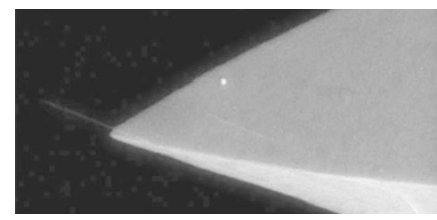

probe tips in many intermittent-contact mode applications. Along with their high aspect ratio, CNT probes are wear-resistant and can be mounted to be perpendicular to the sample within 10 degrees. Typically, nanotube bundles are larger in diameter than a single nanotube, allowing deeper trenches or taller features to be probed. A typical tip is usually $10-30 \mathrm{~nm}$ in diameter and $500 \mathrm{~nm}$ in length, although the diameter of the CNT structure can vary from $5 \mathrm{~nm}$ to $100 \mathrm{~nm}$ and the length can vary from $200 \mathrm{~nm}$ to $5,000 \mathrm{~nm}$.

\section{These notes are compiled in the Nature Materials office} from information provided by the manufacturers. 